\title{
New guidelines for hemorheological laboratory techniques*
}

\author{
Oguz K. Baskurt ${ }^{\mathrm{a}, * *}$, Michel Boynard ${ }^{\mathrm{b}}$, Giles C. Cokelet $^{\mathrm{c}}$, Philippe Connes ${ }^{\mathrm{d}}$, \\ Brian M. Cooke ${ }^{\mathrm{e}}$, Sandro Forconi ${ }^{\mathrm{f}}$, Fulong Liao ${ }^{\mathrm{g}}$, Max R. Hardeman ${ }^{\mathrm{h}}$, Friedrich Jung ${ }^{\mathrm{i}}$, \\ Herbert J. Meiselman ${ }^{\mathrm{c}}$, Gerard Nash ${ }^{\mathrm{j}}$, Norbert Nemeth ${ }^{\mathrm{k}}$, Björn Neu ${ }^{1}$, Bo Sandhagen ${ }^{\mathrm{m}}$, \\ Sehyun Shin ${ }^{\mathrm{n}}$, George Thurston ${ }^{\mathrm{c}}$ and Jean Luc Wautier ${ }^{\mathrm{b}}$ \\ International Expert Panel for Standardization of Hemorheological Methods \\ ${ }^{\mathrm{a}}$ Turkey, ${ }^{\mathrm{b}}$ France, ${ }^{\mathrm{c}}$ USA, ${ }^{\mathrm{d}}$ French West Indies, ${ }^{\mathrm{e}}$ Australia, ${ }^{\mathrm{f}}$ Italy, ${ }^{\mathrm{g}}$ China, ${ }^{\mathrm{h}}$ The Netherlands, ${ }^{\mathrm{i}}$ Germany, \\ ${ }^{\mathrm{j}} U K,{ }^{\mathrm{k}}$ Hungary, ${ }^{1}$ Singapore, ${ }^{\mathrm{m}}$ Sweden, ${ }^{\mathrm{n}}$ Korea
}

\section{Introduction}

This document, supported by both the International Society for Clinical Hemorheology and the European Society for Clinical Hemorheology and Microcirculation, proposes new guidelines for hemorheological methods used in experimental and clinical studies. It is based on a similar document entitled: "Guidelines for measurement of blood viscosity and erythrocyte deformability" published in 1986 by the Expert Panel on Blood Rheology of the International Committee for Standardization in Hematology [45]. Recent methods, techniques and instruments, as well as new approaches to interpretation of results, are added to these new guidelines; wide spread adoption should improve comparability between hemorheological laboratories and increase the reliability of rheological tests.

\section{Collection of blood samples}

\subsection{Sampling time and conditions}

Hemorheological parameters may be affected by the following factors: diurnal variation, level of hydration, recent fluid/food intake, physical activity, drugs and environmental conditions (e.g., temperature, humidity) $[2,15,59,64,76,81]$. These factors should be considered and standardized prior to and during sampling. Since the "wash out" phase of certain drugs may take several weeks, the pharmacodynamics of such agents should also be taken into account. Recent blood transfusions may also affect the results of hemorheological measurements.

\footnotetext{
*Endorsed by International Society for Clinical Hemorheology and European Society for Clinical Hemorheology and Microcirculation.

${ }^{* *}$ Corresponding author: Oguz K. Baskurt, MD, PhD, Professor and Chairman, Department of Physiology, Akdeniz University Faculty of Medicine, Antalya, Turkey. Tel.: +90 242249 6963; Fax: +90 242227 4483; E-mail: baskurt@ akdeniz.edu.tr; URL: http://www.akdeniz.edu.tr/ baskurt.
} 
Sampling should be done after overnight fasting between 08:00 to 10:00 in a quiet environment at normal ambient temperature. Blood withdrawal should be done after a 10 minute resting period and in a seated position. Sampling time and conditions should be described.

\subsection{Sampling procedure}

\subsubsection{Sampling site}

The usual sampling site is an antecubital vein but other sites are possible based upon the specific study protocol [37].

\subsubsection{Tourniquet application}

There is evidence for the significant effects of tourniquet application for blood sampling on the measured hemorheological parameters [35]. The effect of tourniquet application for the localization of a vein and insertion of the needle may continue for several minutes after the removal of the tourniquet [28]. The pressure exerted by the applied tourniquet and the length of tourniquet time may also influence this effect. It has been observed that the hemorheological parameters in samples were less affected if the sampling was done within 90 seconds after the application of tourniquet and without removing it [27] as compared to a procedure where sampling is done after the removal of tourniquet.

\subsubsection{Needles and withdrawal}

If the shear forces to which blood cells are exposed during sampling exceed certain limits cells can be damaged by such forces. The damage may range from minimal alterations in the mechanical properties of red blood cells to hemolysis due to disruption of the cell membrane. Additionally, high shear forces may induce platelet activation and coagulation of plasma, thereby resulting in clot formation in samples and altered levels of some plasma proteins (e.g., fibrinogen) [83]. Using narrow bore needles may increase shear forces during sampling [58]. The second important determinant of the shear forces is the negative pressure applied during withdrawal. Use of commercially available vacuum tubes provides the opportunity to apply a standard negative pressure during sampling.

\subsubsection{Anticoagulants}

Ethylenediaminetetraacetate (EDTA) and heparin are the most widely used anticoagulants in hemorheological studies. The anticoagulant used in hemorheological studies should not dilute the blood and the final concentration should be strictly controlled; the usual levels are $1.5-1.8 \mathrm{mg} / \mathrm{ml}$ EDTA and 14-15 U/ml heparin. Heparin has been demonstrated to influence hemorheological parameters to a greater extent than EDTA [5] and hence EDTA is preferred.

\subsubsection{Recommendation for sampling procedure}

Sampling should be done using sterile procedures, $21 \mathrm{G}$ needles and vacuum tubes containing EDTA. A tourniquet can be used to locate the vein. Sampling should be done as soon as possible after the tourniquet application and, with the tourniquet in place, should be finished within 90 seconds. If possible, vacuum tubes from a single batch should be used within a given study and the manufacturer and batch number of the tubes should be reported. Differences from these conditions should be reported. 


\section{Storage and handling}

\subsection{Storage}

It is well known that hemorheological properties of blood start to change with prolonged storage at room temperature [89]. Although the time course of these changes is slower if the samples are cooled to $4^{\circ} \mathrm{C}$, the safe storage time is still limited [89]. If the measurements can not be performed immediately or within 1 hour after sampling, the samples should be stored at $4^{\circ} \mathrm{C}$ for no longer than 4 hours in order to be useful for most hemorheological parameters The samples should not be frozen. The samples should be warmed to the measurement temperature prior to testing. Note that changes of sample temperature may lead to platelet activation [16], and thus studies specific to platelet aggregation, adhesion or platelet-cell interactions should minimize such changes.

Plasma viscosity is essentially unchanged for 24 hours after the sampling if the blood is left undisturbed, but may increase if the sample is frequently remixed. If the blood will reach the laboratory within six hours, the blood should be sent in its original container. When the specimen reaches the laboratory after six hours and for all postal specimens, the blood should be centrifuged and the plasma harvested. Both whole blood and plasma specimens should be centrifuged at $1,500 \mathrm{~g}$ for $5 \mathrm{~min}$; in the absence of visible clotted material filtration of the plasma is not necessary. If tested on the same day, plasma may be left in contact with the pelletted RBC at room temperature, whereas for an over night delay it is recommended to transfer the plasma to a stoppered container; under sterile conditions it can be stored at room temperature for up to one week.

\subsection{Hematocrit adjustments}

Hematocrit adjustments may be necessary for blood viscosity and red blood cell aggregation measurements. This should be done by adding or removing the calculated amounts of autologous plasma to/from the samples. Plasma should be separated by gentle centrifugation $(1,500 \mathrm{~g}, 5 \mathrm{~min}$ at room temperature). Use of a microcapillary centrifuge is recommended for hematocrit determinations before and after the adjustment.

\subsection{Washing of red blood cells}

All handling of red blood cells (e.g., washing, resuspending) should be kept to a minimum. If the measurement procedures require washing of red blood cells to re-suspend them in a medium different than autologous plasma, this should be done by removing plasma and buffy coat after gentle centrifugation $(1,500 \mathrm{~g}, 5 \mathrm{~min}$ at room temperature). Isotonic phosphate buffered saline (PBS; $290 \pm 10 \mathrm{mOsm} / \mathrm{l}$; $\mathrm{pH}:$ 7.4) is recommended for washing procedure [53]. However, other isotonic buffers can be used and recommended for specific purposes (e.g., Hepes buffer for sickle RBC [54] or for studies where phosphate complexes with specific ions). Repeating the washing procedure two times is usually sufficient. It should be noted that the isotonic media used in washing procedures may influence the properties of blood cells. RBC morphology in the washed samples should be evaluated using a "dilute wet-mount" method: a thin well of grease (e.g., silicone vacuum grease) is applied onto a glass slide, a dilute RBC suspension placed in the well and covered with a glass coverslip, and the RBC quickly viewed in the mid-plane of the well. RBC morphology should not be evaluated by only using a glass slide and coverslip since the two glass surfaces tend to cause crenation (i.e., the "glass slide" effect); the presence of about $0.1 \%$ albumin in the media greatly reduces this artifact. Proper control for any manipulation should always 
be included in the experimental protocol. The washing media and the details of the washing procedure should be reported.

Hemoglobin oxygenation level has been demonstrated to influence RBC aggregation and deformability [90], and thus RBC suspensions for deformability and aggregation measurements should be fully oxygenated. This can be done by rolling the sample for $5 \mathrm{~min}$ in an air-filled test tube whose volume is at least 20 times that of the sample. When exceptions to this requirement for oxygenation are necessary (e.g., studies of sickle RBC at low oxygen tension), the protocol and degree of deoxygenation (i.e., $p_{\mathrm{O}_{2}}$ or saturation) should be indicated.

\subsection{Sample preparation for leukocyte studies}

Leukocytes are quite sensitive to their chemical environment and to the conditions existing during both isolation and measurement. It is desirable, where possible, to carry out analyses using whole or diluted fresh blood to avoid effects of isolation procedures. To limit leukocyte and platelet activation and interaction after withdrawal, theophylline may be added. Neutrophils can be isolated from blood using density gradients, and a number of studies have compared methods for their effects on neutrophil deformability, function or on markers of cell activation [67]. Nevertheless, numerous variants are in use and there is no generally accepted procedure. Media used in any procedure should be endotoxin-free as evidenced by culture-testing. It should be borne in mind that if preparation is carried out in a $\mathrm{Ca}^{++}$ free environment and if neutrophils are suspended in $\mathrm{Ca}^{++}$-free media, spontaneous activation may follow re-introduction of $\mathrm{Ca}^{++}$. The practice of keeping neutrophils cold and then re-warming prior to testing is not recommended because of increased integrin expression and changes in mechanical and adhesive properties upon re-warming [46,67]. In the absence of a standard method, it is recommended that objective evidence of activation be sought (e.g., analysis of morphology or expression of the integrin component $\mathrm{CD} 11 \mathrm{~b}$ compared to values found in whole blood).

\section{Measurement of plasma viscosity, blood viscosity and blood viscoelasticity}

\subsection{Viscometers}

Measurement of blood or plasma viscosity requires the use of viscometers, with the specific details of these instruments dependent upon the fluid being tested. Many blood viscometers are rotational, consisting of two concentric surfaces one of which rotates; their configuration is usually cylinder-incylinder (i.e., Couette) or cone-plate, with the blood sample being sheared in the gap; tube viscometers are also utilized. The shear rate, which is a function of the rotational speed and system geometry, should be nearly constant in the gap. Most rotational viscometers operate at constant shear rates - one of the concentric surfaces is rotated at a constant speed and the shear stress generated is determined from the measured torque and the system geometry. Some rotational viscometers operate at constant shear stresses - one of the surfaces is driven by a constant torque, and the resulting shear rate is determined by measuring the rate of rotation.

The viscosity of blood, more correctly termed the apparent viscosity of blood, is the viscosity of a Newtonian fluid (i.e., a fluid whose viscosity is independent of shear rate or stress) having the same resistance to flow at the same shear rate or shear stress. Thus apparent viscosity is understood to mean its rheological behavior under conditions where the blood is treated as a homogenous fluid and the formed elements (e.g., red blood cells, white blood cells) are tacitly ignored. Data obtained in systems 
with large flow geometries yield macro-rheological properties while small geometries provide microrheological characteristics; in general, data obtained in geometries of $200 \mu \mathrm{m}$ or less are considered micro-rheological $[25,26]$.

The apparent viscosity of blood $\left(\eta_{B}\right)$ is calculated as:

$$
\text { Apparent viscosity }\left(\eta_{B}\right)=\text { shear stress } / \text { shear rate }=(K)(\text { torque }) /(\text { rotational speed }) \text {, }
$$

$K$ is a conversion factor dependent on the geometry of the viscometer system and is determined with the use of Newtonian fluids (e.g., oils of known viscosity) that cover the range of blood viscosity to be measured.

The apparent viscosity of blood can also be measured in tube viscometers by determining the ratio of pressure drop $(P)$ to volume flow rate $(Q)$. The shear stress and shear rate both vary across the cross section of the tube, being zero at the tube center and maximum at the tube wall. The wall shear stress is calculated as:

$$
P R / 2 L
$$

where $R$ is the tube radius and $L$ is the tube length.

The wall shear rate for a Newtonian fluid is:

$$
4 Q / \pi R^{3}
$$

and is higher for non-Newtonian fluids such as blood, but still varies with $Q$. The "effective" apparent viscosity of blood is proportional to the $P / Q$ ratio with a geometric constant $K^{\prime}$ determined using Newtonian fluids. A more rigorous approach is needed to obtain the real wall shear rate and hence the real apparent viscosity at that shear rate. This approach is often termed the Rabinowitsch-MooneyWeissenberg (RMW) method; in general, the RMW method results in a higher wall shear rate and a lower apparent viscosity [63].

The viscometer should ideally be able to operate over a wide range of shear rate or shear stress: for a constant shear rate instrument this should cover from 1 to at least $200 \mathrm{~s}^{-1}$, with an upper range of $300-400 \mathrm{~s}^{-1}$ so that the blood reaches its steady apparent viscosity, while for a constant shear-stress instrument the range should be 0.01-1 Pa. For routine clinical investigations it is recommended that two measurements of apparent viscosity be made, one at each end of the range. Thus a high shear measurement at 200-400 s $\mathrm{s}^{-1}$ and a low shear measurement at $1 \mathrm{~s}^{-1}$ should be made; shear rates approximating these values should be employed if they are not attainable in the instrument used. For a constant shearstress viscometer, the values should be 0.01 and $1 \mathrm{~Pa}$.

Rotational viscometers may have a guard ring to prevent errors due to surface tension artifacts: alternatively, the investigator should assure that surface films artifacts are not affecting results, especially at low shear (see Section 4.4). The components of the viscometer that are in contact with the blood sample should be readily accessible for cleaning following each blood test or disposable components should be used. The surfaces of the container/measuring system of the viscometer should be such that they can be subjected to post-cleaning sterilization with an appropriate disinfectant.

A high degree of instrument sensitivity is required for measuring viscosity at low shear rates: a less than five percent coefficient of variation for a Newtonian fluid of comparable viscosity helps define the lowest measurable shear rate or shear stress. The instrument should be insensitive to the effects of vibration and air drafts or mounted on an anti-vibration table and fitted with a windscreen. Temperature 
control systems (e.g., circulating water baths) should be used to maintain a desired set point. Additional energy input such as direct sunlight should be avoided in order to prevent undesired increases of temperature.

It is usually desirable, especially in follow-up studies, to keep the volume of the blood sample small, and thus the design of the instrument should be such that measurements can be made on small volumes with the sensitivity and accuracy needed, especially at low shear. In rotational instruments, it is important to have precise coaxial alignment of the two surfaces. As mentioned below, RBC syneresis can occur at low shear, thereby yielding a time-dependent torque reading with a rise to a peak followed by a decline. However, if the dynamic response of the torque measuring system is slow, such a peak may not be evident and hence the measured torque will be too low; the investigator should have a knowledge of the dynamic characteristics of the viscometer (i.e. the time required for the torque signal response to reach a steady value both for a standard fluid and for blood samples).

\subsubsection{Steady shear or oscillating shear conditions}

Currently most basic science and clinical hemorheology studies employ one or more steady shear rates to obtain the apparent viscosity of blood; plots of shear stress vs. shear rate or apparent viscosity vs. shear rate are useful for examining the non-Newtonian flow behavior of blood or RBC suspensions. However, there is another approach for examining blood rheology: subject blood to oscillatory or other time-dependent flow. Normal human blood at physiological hematocrits exhibits both a viscous and an elastic response under such conditions and hence exhibits viscoelastic flow behavior. Using this approach it is possible to determine energy stored (i.e., elastic) and energy lost (i.e., viscous) during the deformation. Measurements of the magnitude and phase of sinusoidal oscillatory shear stress and shear rate or shear strain provide direct access to the conventional rheological parameters for a viscoelastic fluid: (1) viscosity and elasticity $\eta^{\prime}-\mathrm{i} \eta^{\prime \prime}$; (2) storage and loss moduli $G^{\prime}+\mathrm{i} G^{\prime \prime}$; (3) Maxwell relaxation time $T_{\mathrm{m}}$. The aggregation tendency and deformability of RBC have a strong influence on the blood viscoelasticity, and hence the shear rate profile reveals the integrated effects of aggregation tendency, aggregate strength and dynamic deformability.

Both the theoretical basis and experimental results of viscoelasticity have been published and an overview of this topic has been presented by Thurston and co-workers [88]. Methods of measurement include use of oscillatory flow in geometries such as cylindrical tubes, Couette cup and bob and cone-plate; non-oscillatory flows have also been used. Each of these methods requires careful consideration of the dynamics, geometry and sensitivity of the testing system, especially since many commercial viscometers are usually designed for industrial material having greater viscous and elastic properties vis-à-vis blood. As with steady shear studies, viscoelastic measurements are subject to the potential measurement problems described below.

\subsection{Measurement of plasma viscosity}

Plasma viscosity is an important factor influencing whole blood viscosity and is directly correlated with the concentration of proteins and lipids [50]; changes in plasma viscosity may occur in both acute and chronic conditions $[9,55,77]$. There are several commercially-available instruments that use different principles and operating techniques (e.g., cone-plate, Couette, rolling ball, tube) but which, if used correctly, yield identical values. Almost all plasmas have Newtonian flow behavior, yet may appear to have shear-dependent viscosity due to surface film artifacts (see Section 4.4). Thus, moderate to high shear rates or pressure gradients are employed in order to minimize or eliminate the surface film artifact. In some rotational viscometers, high shear rates may generate secondary flow resulting in a erroneously 
high values. Temperature control, usually at $37^{\circ} \mathrm{C}$, is necessary since plasma viscosity changes by about $2 \%$ per degree Celsius.

\subsection{Measurement of blood viscosity}

Hematocrit profoundly affects the rheological behavior of blood and RBC suspensions and thus a decision must be made regarding measurements on native, as-drawn blood versus on samples adjusted to a fixed hematocrit. Clinical studies often utilize the native hematocrit since it tends to reflect in vivo conditions, whereas studies designed to evaluate RBC aggregation and deformability by viscometry require hematocrit adjustment to allow comparisons.

\subsubsection{Measurement temperature}

Blood viscosity varies inversely with temperature and viscometers must be capable of maintaining the sample temperature constant at the desired temperature with a maximum error of $\pm 0.5^{\circ} \mathrm{C}$. Pre-warming of the blood sample and instrument sample container to the working temperature is suggested. When using an external water bath with a circulator, the connecting tubing should be sufficiently short and insulated to insure that the temperature of the blood sample itself is at the intended level. A direct measurement of the working temperature of the test sample is a desirable instrument feature. It is recommended that blood viscosity measurements be made at $37^{\circ} \mathrm{C}$. Measurements at lower temperatures may be required for the study of some patients (e.g., Raynaud's phenomenon) or for studies specific to temperature effects.

\subsubsection{Measurement technique}

The blood sample container and measuring system should be clean and dry prior to each measurement. RBC aggregation at low shear may cause erythrocyte syneresis and sedimentation, and thus at low shear rates the blood sample must be well-mixed immediately prior to each shear rate measurement. It is generally not acceptable to use a scanning method in which shear is progressively and continuously increased or decreased since at lower shear rates there may be insufficient mixing: without adequate mixing the results are invalid. Any deviation from this approach should be stated when reporting results. Note that RBC aggregation and the attendant problems of low-shear viscometry are often enhanced in pathological samples, and thus erythrocyte sedimentation may present a problem especially in a tube viscometer.

Viscosity results for native whole blood samples from patients should be compared with those from normal, gender and aged matched individuals made at the same shear rate and temperature. If it is desired to assess whether any change in apparent viscosity is attributable to factors other than hematocrit, the patient's blood viscosity should be compared with the results obtained in normal blood at a comparable hematocrit. For this latter comparison each laboratory may prepare its own reference range relating apparent blood viscosity to hematocrit using normal blood whose hematocrit is adjusted to cover a $20-70 \%$ range. As a quality control check, reference ranges should be re-determined periodically in each laboratory.

\subsection{Potential measurement problems}

It is necessary to consider some difficulties and potential artifacts associated with rheological measurements on blood, plasma and related fluids. Three properties of these materials can lead to problems in measuring rheological behavior: (1) the plasma proteins are surfactants and form a protein layer or film at fluid-air interfaces; (2) the erythrocytes are denser than plasma; (3) at low shear rates, RBC tend to aggregate. 


\subsubsection{Surfactant layer}

The surfactant layer formed by plasma proteins at the fluid-air interface has mechanical strength and is a semi-rigid film. Consequently, the pressure drop across such interfaces, as usually exists in a capillary viscometer, is not zero. The surfactant layer can also transmit significant torque to the torque-measuring element in rotational viscometers. In general, this effect is important only for plasma and serum or for low-hematocrit RBC-plasma or RBC-serum suspensions; at normal or high hematocrits, the additional torque is only a small percent of the total and can be essentially ignored. However, an unfortunate result of this additional torque is the artifact of non-Newtonian flow behavior for normal human plasma. Procedures to minimize or eliminate the influence of the protein layer depend on the type of viscometer used: for capillary viscometers, use fluid filled pressure transducers that connect to sample reservoirs below the fluid-air interfaces, or obtain pressure drop and flow rate data using two capillaries of the same diameter but significantly different lengths. In the case of rotational viscometers, one approach is to insert a "guard" ring through the interface, thereby isolating the torque-sensing element from the film-associated drag. Note, however, that the guard ring must rotate at the same angular velocity as the torque-measuring element of the viscometer, and is thus not useful for viscometers in which the element both rotates and senses torque. Note also that the need to employ these approaches will depend on the details of the viscometer design and may not always need to be utilized.

\subsubsection{Red blood cell sedimentation}

RBC sedimentation is slow for individual cells, and for an individual RBC in plasma is only about $0.13 \mu \mathrm{m} / \mathrm{min}$ or $0.08 \mathrm{~mm} / \mathrm{h}$. If the suspending medium does not promote marked RBC aggregation, and if the hematocrit is $15 \%$ or greater, the presence of neighboring red cells causes the settling to be hindered and hence slower. RBC aggregation greatly increases the sedimentation rate since the settling rate of particles is dependent on the square of the particle size. A typical, steady, hindered settling rate for $40 \%$ hematocrit normal human blood is about $200 \mu \mathrm{m} / \mathrm{min}$ or $10 \mathrm{~mm} / \mathrm{h}$. Usually, sedimentation in viscometers is not a significant problem if the wall shear rate is above several inverse seconds. However, at lower shear rates, the effect of erythrocyte sedimentation must be considered, especially for horizontal tube and cone-and-plate viscometers. For example, in cone-plate viscometers a cell-poor, lower viscosity layer can develop at the upper element; if torque is sensed by this upper element then torque readings and hence calculated viscosity values are too low. RBC aggregation may be greatly enhanced in certain pathologic states (e.g., diabetes, myocardial infarction), thus making this process of particular importance when measuring such samples [61].

\subsubsection{Red blood cell syneresis}

In addition to sedimentation, RBC aggregation also causes red cell syneresis, which is the partial separation of RBC from their suspending medium as they aggregate. This process leaves layers of cell-poor plasma at the walls of viscometers of all types. If using a concentric cylinder viscometer filled with wellmixed and well-stirred blood, the effects of syneresis are seen very soon after starting the viscometer at a constant low shear rate of about $2 \mathrm{~s}^{-1}$ or less. In this case, the torque first rises from zero to a peak, then decays in an exponential manner with time, finally reaching a steady value after approximately 30 minutes. This time-dependent torque response is due to the startup of the fluid motion at very short times, syneresis of the erythrocytes away from the viscometer surfaces at all times, and finally, at very long times, sedimentation effects. Note that essentially all investigators agree that using the final steadystate value of torque is incorrect since this value is the result of fully completed syneresis plus some variable degree of sedimentation: its use results in shear stress and viscosity values that are too low. Two different methods have been proposed for assigning the correct torque at shear rates below about $2 \mathrm{~s}^{-1}$ : 
one uses the peak torque value while the other extrapolates the exponentially decaying torque data back to zero time. Using normal blood the two methods diverge at shear rates at and below $1 \mathrm{~s}^{-1}$, with the peak method often leading to an artifact of Newtonian flow at very low shear; the results are usually coincident at higher shear. Note that syneresis can also affect vertical tube viscometers at low shear rates, in that a cell-poor layer forms at the tube wall thereby decreasing resistance to flow, whereas if the viscometer tube is horizontal $\mathrm{RBC}$ sedimentation tends to increase flow resistance.

\subsection{Expression of results}

Values for apparent blood viscosity at $37^{\circ} \mathrm{C}$ should be given in milliPascal seconds (mPa s), which is equal to the older term "centiPoise" (cP), at a stated shear rate (or shear stress) and hematocrit. For clinical purposes, the laboratory reference range at $37^{\circ} \mathrm{C}$ should be given for each of the shear rates (or shear stresses) reported.

\section{Red blood cell aggregation}

Under static conditions, RBC in normal human blood form loose aggregates having a characteristic face-to-face morphology similar to a stack of coins; such aggregation is frequently referred to as rouleaux formation [70,75]. Moreover, given sufficient time and a non-confining space, individual rouleaux can cluster, thereby forming three-dimensional structures. The attractive forces involved are relatively weak and aggregates can be dispersed by applied shear and during flow. This process is reversible so that aggregates reform under quiescent conditions. RBC aggregation increases low shear blood viscosity and affects micro-vascular flow dynamics [48] and can be markedly enhanced in several clinical states.

Factors affecting RBC aggregation can be divided into intrinsic and extrinsic factors: (1) Extrinsic factors include levels of plasma proteins (e.g., fibrinogen, macroglobulins), hematocrit and shear rate; (2) Intrinsic factors include RBC shape, deformability and membrane surface properties. Extreme changes from the normal biconcave shape and/or decreased cellular deformability will decrease aggregation. RBC surface properties and structure, including surface charge and the ability of macromolecules to penetrate the membrane glycocalyx, greatly affect aggregation for cells suspended in a defined medium (e.g., compatible plasmas, polymer solutions). Thus, RBC cellular properties determine the cell's intrinsic tendency to aggregate and are the basis for the term "RBC aggregability". Aggregability is increased if, for the same suspending medium, the test RBC aggregate more than control cells or cells from a different blood donor; the converse finding indicates decreased aggregability.

\subsection{Methods}

Various approaches and methods have been employed to assess RBC aggregation, and there are currently commercial instruments with the ability to provide information relevant to one or more aspects of aggregation. However, there are no "standard" or "calibrator" suspensions for RBC aggregation and thus each method may yield one or more parameters that are possibly unrelated to those obtained by another technique. 


\subsubsection{Measurement of light transmission or light reflection through/from RBC suspensions}

This technique is based upon the increase of light transmission (LT) through [14] or decreased light reflection (LR) from [41] a RBC suspension that occurs when individual cells aggregate into rouleaux or rouleaux-rouleaux complexes; gaps in the suspending medium between the aggregates allow more light to pass through the RBC suspension or less light to be reflected. Recording of LT or LR for further analysis is often termed syllectometry. Devices that employ this approach usually use a transparent coneplate system, parallel rotating surface(s), Couette or a rectangular flow chamber geometry.

Different indices of aggregation can be measured based on both light transmittance and reflectance:

(a) RBC aggregation at stasis: A blood sample is first briefly sheared at a constant high shear rate, usually in the range of several hundred $\mathrm{s}^{-1}$, to disperse all pre-existing aggregates. The shear rate is then abruptly decreased to zero following which light intensity is integrated for a fixed time, usually in the range of a few seconds or minutes. The numeric value resulting from this integration serves as one measure of aggregation, with its absolute value dependent on both aggregation tendency and integration time. Other indices that may be calculated using the recorded time course of LT or LR can be found elsewhere $[14,41]$.

(b) RBC aggregation at low shear: The blood sample is first briefly sheared to disperse all pre-existing aggregates, following which the shear rate is abruptly reduced to a low fixed shear rate (e.g., $3 \mathrm{~s}^{-1}$ ) and the light is integrated over a fixed time. The numeric value resulting from this integration is again a measure of aggregation. Note that this method reflects the extent of aggregation under conditions at which low fluid shear promotes cell-cell contact. The rationale for the low shear measurement evolved from earlier studies in which a "flow to stasis aggregation ratio" (FSAR) was calculated (i.e., ratio of the extent of aggregation at low shear to that at stasis [91]).

(c) RBC aggregation kinetics: Several kinetic parameters can be extracted from digital recordings of the time course of LT or LR, including two exponential time constants and an overall half-time for aggregation.

(d) RBC disaggregation shear rate or stress: The shear rate just sufficient to disperse existing aggregates and to prevent aggregate formation, without causing cell deformation, is determined by applying different shear rates to a blood sample and recording steady-state LT or LR as a function of applied shear rate. LT is greater at low shear due to aggregate formation and also greater at high shear due to cell deformation; LR is opposite in behavior (i.e., decreased with increased aggregation). The resulting data are fitted by non-linear regression analysis, with the disaggregation shear rate determined as the shear rate with minimal LT or maximal LR; this shear rate is sometimes referred to as the threshold shear rate. The RBC disaggregation shear stress can be obtained by multiplying the disaggregation shear rate by the viscosity of the blood sample measured at the same shear rate.

\subsubsection{Microscopic indices of red blood cell aggregation}

Microscopic aggregation indices are direct methods for assessing aggregation at stasis and at low flow [4]. For example, a dilute RBC suspension (e.g., $\approx 1 \%$ hematocrit) of known cell count is placed in a standard hemocytometer chamber and left undisturbed for a defined period (e.g., 5 or $10 \mathrm{~min}$ ). Several areas of the chamber are then recorded by video microscopy following which the number of cellular units (i.e., a single mono-dispersed cell or a single cellular aggregate) per unit volume is counted. The microscopic aggregation index is then calculated as the ratio of (total cell count per volume)/(number of cellular units per volume), and increases with the extent of aggregation. In addition, the geometry of the aggregates can be assessed for perimeter, area and several shape factors. RBC aggregation can also 
be investigated by microscopic observations under various flow conditions (e.g., parallel plate, counterrotating surfaces) [4]. These methods can be combined with computerized image analysis methods to improve the accuracy and efficiency of data collection.

\subsubsection{Erythrocyte sedimentation rate}

The sedimentation behavior of RBC suspensions in defined vertical circular geometries has been used for decades as an index of health and of the presence of plasma proteins which promote RBC aggregation. In very dilute suspensions, the rate of a spherical particle's sedimentation increases with the square of its radius (i.e., Stokes' settling). This concept has been extended to the crowded environment of blood or RBC suspensions such that an increased settling rate or extent is associated with increased RBC aggregation. Techniques to determine the erythrocyte sedimentation rate (ESR) include the Wintrobe and Westergren methods in which the distance fallen by the cells is determined at the end of a 60 min period of settling. Either manual or automated methods may be used to obtain additional detailed information about the process, including the time required to initiate settling and the true steady-state rate of settling; ultrasound methods have also been employed to follow the settling process.

\subsubsection{Low shear viscosity}

In general, blood viscosity at shear rates below about one to five inverse seconds increases with RBC aggregation, and thus low shear apparent viscosity is used as an index to the intensity of aggregation; the ratio of low to high shear viscosity has also been utilized. Techniques and precautions for low shear blood viscosity measurements are discussed in Section 4.4. It is important to note a potential artifact possible with this method: small decreases of RBC deformability can increase low shear viscosity, even for RBC in plain buffer, but may not affect high shear results [11]. Given the unaltered high shear viscosity and elevated low shear viscosity, one could incorrectly infer enhanced RBC aggregation.

\subsubsection{Ultrasound backscattering}

Individual RBC and RBC aggregates behave as ultrasound scattering objects, with the extent of backscattering related to the volume of the cell or aggregate. The backscattering coefficient is commonly used to characterize ultrasound scattering by blood and RBC suspensions, with the magnitude of the coefficient increasing with the degree of aggregation; for aggregating suspensions, the coefficient decreases with increasing shear rate [23].

\subsection{Measurement conditions}

Note that all current techniques used to measure RBC aggregation are sensitive to hematocrit and the properties of the suspending medium (e.g., density, viscosity) as well as to the levels of pro-aggregating plasma proteins: (1) light transmission and reflection methods have a biphasic response to hematocrit with some indices decreasing with increased or decreased hematocrit; (2) microscopic methods are unreliable at hematocrit extremes; (3) ESR varies inversely with hematocrit and is decreased by increased medium density and viscosity; (4) low shear viscosity is extremely sensitive to hematocrit. Thus, while measurements using native blood may be potentially useful for some clinical evaluations or for largescale population studies, experimental studies intended to specifically measure RBC aggregation should utilize RBC suspensions adjusted to a fixed hematocrit. Either $40 \%$ or $45 \%$ are recommended, except for microscope-based methods which require a lower hematocrit. Aggregation studies for RBC suspended in various polymer or protein solutions should substitute the solution for buffer during the second wash. For the measurement of RBC aggregability it is recommended to use fixed concentrations of dextran (e.g., $3 \mathrm{mg} / \mathrm{ml} 70 \mathrm{kDa}, 0.5 \mathrm{mg} / \mathrm{ml} 500 \mathrm{kDa}$ ), but other polymers may also be suitable. If available, the 
ratio of weight-average to number-average molecular weight (i.e., $M_{\mathrm{w}} / M_{\mathrm{n}}$ ) for the polymer fraction should be stated as well as the viscosity of the polymer solution.

\subsection{Precautions}

It is important to recognize the possibility of potential artifacts or challenges associated with various methods. Some of these include: (1) Assuming that a shear-based method applies sufficient shear stress to initially fully disperse all aggregates for suspensions having very high aggregation, since failure to disperse pre-existing aggregates usually results in falsely low indices; (2) Assuming that elevated low shear viscosity always indicates increased aggregation, since small decreases of RBC deformability will increase low shear viscosity, even for RBC in simple buffer, but may not affect high shear results; (3) Increased suspending phase viscosity due to greatly increased levels of plasma proteins (e.g., macroglobulins) or to added polymers will tend to decrease the RBC sedimentation rate, retard the kinetics of RBC aggregation, and increase fluid shear stress at a given shear rate; (4) The morphology of RBC aggregates may differ depending on the polymer or protein causing aggregation, with macroglobulins and some large polymers resulting in the formation of clumps rather than face-to-face rouleaux [84].

The extent to which any or all of the abovementioned potential problems affect a specific method should be considered. Thus the following procedures are recommended to validate aggregation results: (1) Examine RBC morphology in the suspending medium to determine alterations from the normal biconcave shape (e.g., crenated, cupped); (2) Examine the morphology of aggregates to determine their shape (e.g., rouleaux, clumped, smooth spheres), with clumped or spherical forms indicating intense aggregation and the possible need for higher than normal stress levels to disperse such aggregates; (3) As practical, determine light transmission or reflection from a RBC suspension as a function of increasing dis-aggregating shear rate in order to assure that this rate is sufficient to fully disperse aggregates.

\subsection{Interpretation of the results}

Parameters reflecting the extent of RBC aggregation are usually dimensionless and are thus usually not comparable between instruments, but within an experimental study using a single method, comparisons are most likely valid if the hematocrit is adjusted to a constant value. However, kinetic indices of RBC aggregation such as half-time of aggregation have standard dimensions (e.g., seconds) and therefore should be comparable. Note that it is now clearly evident that donor-to-donor differences of "cellular factors" can affect aggregation, even for normal RBC in a defined aggregating medium (i.e., RBC "aggregability"). Thus variations of results for a given experimental protocol may reflect biological variability as well as the characteristics and precision of a particular instrument.

\section{Measurement of red blood cell deformability}

The ability of RBC to deform in response to an applied force (i.e., RBC deformability) is required for their normal passage through the capillaries of the microcirculation and the splenic sinusoids. Erythrocyte deformability also influences blood viscosity in wide-bore vessels. The high deformability of the normal erythrocyte is a consequence of its low intracellular (cytoplasm) viscosity, its excess of surface membrane area in relation to cell volume (S/V ratio) and the viscoelastic properties of its cell membrane. Loss of deformability may arise from impairment of any one, or a combination, of these properties. Similarly, the methods and instruments available for measuring erythrocyte deformability may be sensitive 
to these properties to differing degrees. For measurement of their deformability, erythrocyte suspensions can either be sheared in large geometry systems (e.g. viscometry or laser diffraction ellipsometry, also called ektacytometry) or made to traverse narrow channels (e.g. micro pipettes, micro channels or filter pores).

\subsection{Methods}

\subsubsection{Bulk filtration methods}

Erythrocyte filtration methods were previously the most widely utilized approach to the assessment of RBC deformability. Because of the apparent simplicity of the method many variations have been developed. In all cases the ability of erythrocytes to pass a filter is measured, either by the time required for passage of a certain volume of erythrocyte suspension or by the pressure-flow relationship. Polycarbonate membranes with a pore diameter of 3-5 $\mu \mathrm{m}$ were usually used. The geometric characteristics of the filter, especially the mean pore diameter, the total number of pores and their distribution across the membrane surface, are of critical importance for the final outcome. It is therefore essential to perform comparable filtration measurements of different samples with the same batch of membranes. If possible, it is preferred to use the same membrane that is cleaned between measurements. Note that since filtration results are functional assays and can be affected by many factors, it is recommended to use the term filterability for these measurements $[39,40]$.

The various filtration systems used gravity, positive pressure or negative pressure driven filtration. A major disadvantage of these bulk filtration systems is that due to the relatively high stresses employed, minor deformability changes often remain undetected. In addition, pore blockage by the more rigid leukocytes, platelet micro aggregates or even rigid erythrocytes can be a complicating factor [74]. Further, techniques used for the preparation of a pure erythrocyte suspension carry the inherent risk that RBC sub-populations may be selectively removed. Changes in filterability could result from a change in deformability of either the whole cell population or a subpopulation. Finally, intrinsic erythrocyte factors (e.g., MCV for smaller diameter pores, MCHC for larger diameter pores), and cell morphology may affect the final outcome.

\subsubsection{Pore transit analysis}

Passage of individual RBC through individual cylindrical pores can be monitored by recording the electrical conductance across a special filter containing only about 30 pores of well-defined geometry [56]. Dilute RBC suspensions are filtered through these "oligopore filters" using only a few centimetres of water pressure gradient. The resulting resistive pulses are digitally recorded and analyzed to obtain several parameters reflecting various aspects of RBC passage through the pores [6]. Filters with different pore sizes can be used and the cell size-pore size ratio determines the sensitivity of the method to alterations in deformability [8]. While offering the ability to detect subpopulations of cells with differing deformability, commercial versions of this technology are, unfortunately, not currently available.

\subsubsection{Laser diffraction ellipsometry or ektacytometry}

This technique is based on shear-induced elongation or deformation of RBC into ellipsoid-like shapes. Shear stress is applied to a dilute erythrocyte suspension by a rotational cylindrical Couette or plate-plate system, by flow between parallel plates or by tube flow. Regardless of the geometry employed, the shear stress acting on the cells is the product of local shear rate times the suspending medium viscosity. A laser beam, passing through the cell suspension at a right angle relative to the flow vector, is diffracted by the cells. The pattern of diffracted light is analyzed either via measuring light levels at defined locations or 
by image analysis. The results are commonly expressed as an elongation index (EI) or a deformation index (DI): both reflect the aspect ratio of the ellipse as (length - width)/(length + width) and increase with cell deformation.

By subjecting normal erythrocytes to a progressive increase in shear stress, an S-shaped deformation curve (i.e., EI versus shear stress) is obtained. Additional specialized testing methods include: (1) for mainly diagnostic purposes, RBC elongation, at a constant high shear stress, is measured while the osmolality of the suspending medium is progressively increased from $\sim 100$ to $450 \mathrm{mOsmol} / \mathrm{kg}$, generating an EI-osmolality pattern often referred to as an osmoscan [82]; (2) continued exposure of either $\mathrm{RBC}$ or RBC ghosts to high shear stress can be used as an erythrocyte stress test, mainly reflecting cell membrane fragility. The latter effect for RBC can be analyzed by measuring EI continuously during the stress experiment [20] or by comparing cell deformability before and after the application of shear stress [20,57]. Studies using resealed RBC ghosts involve measuring EI over time at constant stress to study membrane fragmentation and thus membrane stability [20].

\subsubsection{Micropipette aspiration}

Since its development in the early 1950s, micropipette aspiration of erythrocytes has been a useful experimental technique to provide precise, quantitative information on the rheological properties of individual erythrocytes. Several uses of micropipettes are possible and include: (1) measuring membrane elastic shear modulus and area compressibility modulus by partial aspiration of a small portion of membrane into 1-2 $\mu \mathrm{m}$ pipettes; (2) measurement of overall cell deformability by aspirating the entire cell into 3-5 $\mu \mathrm{m}$ pipettes; (3) determining the time constant for RBC shape recovery, and hence membrane surface viscosity, by extending then releasing a cell point-attached to a surface; (4) determination of cell volume and membrane surface area by aspirating cells into small pipette to form a spherical outer portion and a tongue within the pipette. However, such measurements on single erythrocytes are tedious, time consuming and require a high degree of technical skill. Thus, while micropipette methods are invaluable for basic science studies, the technique has remained of limited clinical value.

\subsubsection{New and emerging single cell techniques: micro flow devices, optical traps, stretchers and tweezers}

Recent advances in nanotechnology, molecular biomechanics and micro flow techniques have led to the development of a new suite of techniques for quantifying erythrocyte deformability, including optical traps, stretchers and tweezers. Micro flow channels allow real-time mechanical manipulation and analysis of single erythrocytes at the micro- and nanometer level and with nano- and pico-Newton force resolution. At present, however, these techniques are still highly experimental and precise control and measurement of flow (i.e., shear stress) in such miniature devices presents a problem.

\subsubsection{Viscometry, Tk factor calculation}

This bulk viscosity method for estimating deformability was cited in the prior Guidelines. However, the Tk parameter is also affected by plasma viscosity and hematocrit, and thus is not currently recommended for erythrocyte deformability assays.

\subsection{Measurement conditions for ektacytometry}

Ektacytometry is currently the most widely used technique for the measurement of RBC deformability; measurement conditions, precautions, expression of results and interpretation issues for this method are discussed below. 
Based upon many literature reports, several experimental conditions are required in order to avoid measurement problems: (1) Dilution of whole blood or cell suspensions in isotonic solutions of polyvinylpyrrolidone (PVP) or dextran in buffer to achieve a hematocrit of about $0.2-0.3 \%$; (2) Use of a PVP or dextran medium having a viscosity of about $30 \mathrm{mPa} s$ at $37^{\circ} \mathrm{C}$ since below this level cell deformation at constant shear stress is affected by medium viscosity; (3) Measurement at $37^{\circ} \mathrm{C}$ since it has been observed that a given impairment in erythrocyte deformability, under certain experimental conditions, could only be detected at $37^{\circ} \mathrm{C}[10]$.

The shear stress range to be used depends on the ultimate goal of the measurements: (1) For comparisons of EI measured at specific levels of shear stress measurements should be done in the mid-range (e.g., 3 Pa); (2) If the EI-SS curve will be parametrized, SS range should be extended to cover the level sufficient to reach the maximum EI (see Section 6.3).

\subsection{Expression of results}

Deformation data as an elongation index (EI) are usually presented graphically as a function of shear stress, with EI having a value for normal cells of about 0.6 at $50 \mathrm{~Pa}$. Tabular results at one or more selected stress levels are also appropriate. EI values taken from deformation curve at about $3 \mathrm{~Pa}$ are more sensitive for changes in deformability but have also a higher CV than values at higher levels (e.g., $50 \mathrm{~Pa}$ ). In order to define one or more global parameters of deformability, the entire data set can be parameterized. Two approaches have been described to obtain the maximal deformation at infinite shear (EI $\max )$ and the shear stress at one-half of this maximum $\left(\mathrm{SS}_{1 / 2}\right)$ : (1) by empirical non-linear curve fitting [17]; (2) by linear regression using a method analogous to a Lineweaver-Burk plot (i.e., inverse of shear stress versus the inverse of EI) [12]. The upper value of SS used for measurements should not be below $50 \mathrm{~Pa}$ for such calculations. Osmotic scan results are expressed by several osmolality values defined by the deformation-osmolality curve: hypo- $\left(\mathrm{O}_{\min }\right)$, iso- $\left(\mathrm{O}_{\max }\right)$ and hyper- $\left(\mathrm{O}_{\text {hyper }}\right)$ osmotic conditions [22]. Stress test results for intact RBC report deformability prior to and after a period of high stress, with membrane fragility (i.e., fragmentation) for ghosts expressed as the half time for decay from maximal deformation during the application of a given stress

\subsection{Interpretation of results}

While the range of EI values for patients and healthy individuals is usually relatively small, at least in the presence of a functioning spleen [7], there are results indicting the clinical potential of assessing RBC deformability by ektacytometry [43]. In addition to statistical analysis of data at selected shear stress levels, other approaches are possible: (1) examining the initial slope and the deformation index at high stress, since the slope primarily reflects membrane mechanical characteristics while the high stress value is mainly related to the cell's surface area to volume ratio and to intracellular viscosity or MCHC [21]. Interestingly, deformation values at isotonic conditions are relatively insensitive to cell volume over a range of MCV [42]; (2) using the two parameters obtained from either one of the two curve fitting methods (see Section 6.3).

All ektacytometric techniques described have in common that the average deformability of all erythrocytes in the test sample is measured. However, in the presence of very rigid cells (e.g., chemically fixed discocytes, cells from subjects with sickle cell disease), the diffraction image has a central circular component superimposed on the normal ellipsoid shape; estimation of the EI is thus adversely affected. As an alternative to testing sub-fractions of the RBC population, a deconvolution approach has been 
described [86]. When a detailed cell-by-cell analysis is required (e.g., infected RBC in malaria), direct observation in defined shear fields is possible using microscopy plus transparent shearing systems such as a counter-rotating rheoscope [32] or cells attached to a surface in a flow chamber [78].

\section{White blood cell rheology}

The rheological factors influencing the circulation of white blood cells (i.e., leukocytes) can be broadly divided into: (1) resistance to deformation in response to hemodynamic forces; (2) adhesiveness to the vessel wall. Both are influenced by the activation of leukocytes in response to physiological and pathological stimuli, and changes in both can cause vascular obstruction and damage such as seen in vascular inflammatory disorders. The rheology of neutrophils has been studied more widely than rheology of other leukocyte subsets. An important caveat is that the activation state and hence the rheological properties of neutrophils are highly sensitive to preparative procedures. At the same time, it is changes in this state that are of interest and likely to be reflected in clinical measurements. Lymphocytes appear less labile in their behavior, and while monocytes are highly reactive, rheological studies on these cells have been rare. Much of what follows relates to neutrophils, but the measurement systems and principles should apply to other subsets. Methods for preparing samples are considered in Section 3.4; this section concentrates on analysis of resistance to deformation and adhesiveness.

\subsection{Measurement of leukocyte deformability and related parameters}

\subsubsection{Suspension filtration}

Filtration techniques have been the most readily available and widely used methods for testing resistance to flow of leukocytes, and have been used in a range of clinical studies (see [47,65] for reviews). Use of whole or diluted blood is possible with some filtration systems [29]. In the case of isolated neutrophils, results correlate with morphological activation [66] or are sensitive to agents which induce activation [67].

In general, the flow rate is measured for a cell suspension or blood suspension passing through a filter with pores of $5 \mu \mathrm{m}$ or $8 \mu \mathrm{m}$ diameter under the action of a constant driving pressure [47,65]. Alternatively, a constant flow rate may be used, and pressure monitored. Flow-versus-time may be used to assess achievement of a steady state and the tendency to block pores, reflecting the flow resistance of the main population or a resistive sub-population. Mathematical models of the flow curves may allow detailed analysis of sub-populations of the flowing leukocytes [29,68]. In principle, filtration methods do not necessarily distinguish between adhesive properties, geometric properties and cytoplasmic properties, or clearly identify the contribution of recognizable sub-populations. The use of $\mathrm{Ca}^{++}$-free media should, however, minimize contributions from adhesion.

\subsubsection{Pore transit analysis}

Modified filtration techniques can measure the properties of individual cells, rather than population averages, by using dilute suspensions and electronic sensing of transit through special polycarbonate filters termed oligopore filters (see Section 6.1.2) [73]. The time taken for individual leukocytes to pass through $8 \mu \mathrm{m}$ diameter pores is measured under constant pressure [73]. This approach has the advantage that a frequency distribution of cell properties is attained for about 1000 cells and there is the potential to detect abnormal sub-populations of cells, although trapped cells and some very slow-flowing cells may not be detected [19]. 


\subsubsection{Micromanipulation}

Detailed information on individual leukocytes can be obtained by micromanipulation $[19,34,69]$. Structural viscoelasticity may be tested by partial aspiration into a micropipette with a diameter about $2 \mu \mathrm{m}$ [34], or the overall resistance of a whole cell to entry into 4-8 $\mu \mathrm{m}$ pipettes can be measured [69]. Few clinically-related studies have used this complex and time-consuming methodology. Activated neutrophils are, however, clearly more rigid and the effects of specific pathological agents may be studied [19]. In these methods, cation chelation to abolish adhesion via receptors described to date is essential.

\subsubsection{Formation of pseudopodia and F-actin}

With activation of neutrophils, globular G-actin is rapidly converted to filamentous F-actin, but the F-actin content then decreases partway towards its initial level [44]. At the same time, pseudopodia form. Morphological examination of fixed neutrophils can be used to assess the proportion which are activated (i.e., the percentage with distorted instead of smooth spherical shapes). Cell shape can be quantitated using: (1) isolated neutrophils [66]; (2) stained blood films [60]; (3) whole blood fixed relatively mildly, followed by lysis of red cells [62]. The percentage of morphologically active cells has been found to correlate strongly with impaired filterability for purified cell suspensions [66]. F-actin content can be measured by quantifying the binding of fluorescent phalloidin to F-actin [44]. However, while actin polymers clearly contribute to changes in neutrophil rigidity, there may not be an exact correlation between F-actin level and overall cell deformability [73].

\subsection{Measurement of leukocyte adhesion}

\subsubsection{Surface attachment}

Adhesion of isolated leukocytes can be tested by settling them onto surfaces coated with receptor proteins or with cultured endothelial cells [18]. The incubation time prior to washing off non-adherent cells need only be of the order of minutes. Quantification of adhesion can be done by direct microscopic counting of the cells or by using leukocytes loaded with dyes and the dye quantified; use of radio-labeling is no longer popular, and fluorescent dyes are more commonly used.

Monitoring the adhesive behavior of neutrophils may be based on their capability to attach to selectins, adhesion molecules of the immunoglobulin superfamily, to purified proteins from plasma (e.g., albumin or fibrinogen) or to proteins found in the extracellular matrix such as collagen, laminin, fibronectin. Concentrations of these agents that are used to coat a surface are typically of the order of microgram per ml. Binding to selectins should be efficient for unstimulated neutrophils, while the other receptors all bind specific integrins which generally require conformational activation to bind efficiently. The limiting factors are thus expression of selectin ligands which tend to decrease on activation and neutrophil activation that increases integrin binding. Cultured endothelial cells which have been stimulated with cytokines such as tumor necrosis factor- $\alpha$ or interleukin- $1 \beta$ present selectins and integrin-ligands as well as activating agents.

To distinguish clearly between contributions from selectin-mediated and integrin-mediated adhesion when both are possible, or to test the influence of fluid forces on adhesion, flow-based assays are required. In flow, selectins support rolling adhesion, while integrins support immobilization. Various forms of flow chambers have been described for flow assays, and flow rates are chosen to mimic conditions in post-capillary venules where neutrophil recruitment occurs in vivo. Whole blood has rarely been used in adhesion assays. In flow-based assays with blood, adhesion depends on hematocrit and red cell aggregation [1]. It may therefore be difficult to separate the effects of the hemorheological variables from the adhesive properties of the leukocytes themselves. 
Because of difficulties in obtaining blood borne leukocytes or isolation problems, and difficulties in obtaining endothelial cells for adhesion studies, cell lines may be used to investigate the roles of specific receptors. Erythroid K562 or leukemic HL60 cell lines can be conditioned to express adhesion molecules and can be used to explore the molecules involved in cell-cell adhesion. Chemically or genetically modified cell lines can be used in flow systems to further investigate the molecular alterations responsible for increased adhesion to endothelial cells or to coated surfaces [92]. For adhesion studies of specific receptors on endothelium, genetically modified cells lines, expressing only the receptor of interest, can be used as a substrate for leukocyte adhesion.

\subsubsection{Leukocyte aggregation}

A technically straight-forward measurement is to quantitate cell-cell aggregation, usually neutrophilneutrophil but neutrophil-platelet aggregation can also be studied. Neutrophil aggregation can be measured in whole blood or in isolated preparations by cell counting techniques [30,52]. Immunofluorescence and flow cytometry can be used with whole blood to detect aggregates which bear fluorescentlylabeled antigens characteristic of neutrophils and platelets [80]. Leukocyte-platelet aggregation has been found to be elevated in several clinical conditions including, for instance, post-cardio-pulmonary bypass [79].

\subsubsection{Expression of surface adhesion receptors}

Since adhesion of neutrophils depends on surface-presented integrins, L-selectin or selectin ligands, measurement of the expression of these receptors using fluorescently-labeled antibodies and flow cytometry may be a surrogate for an adhesion assay. Measurement of the level of surface expression of integrins CD11b and CD18 can be used to monitor adhesive potential [13]. After cell activation, CD11b rises more than CD18. In addition, the method can be applied to whole blood for clinical studies, where expression may be lower than on isolated cells because of the lack of preparative trauma [72]. However, it may be noted that the conformation of integrins is important in defining adhesive potential. Antibodies specifically binding to epitopes exposed on activated integrins are available [31], and may be used to quantify active receptors.

Since it is known that L-selectin is shed from neutrophils upon activation, reduction in expression should also indicate activation [71]. In fact, plasma contains a high level of the presumably-shed molecule, and an alternative approach to measure $\mathrm{CD} 11 \mathrm{~b}$ on neutrophils has been to detect the level of soluble L-selectin in plasma. Increasing concentrations of L-selectin due to shedding have been shown to correlate with positive prognosis in septic shock [33].

\subsection{Precautions and interpretation of data}

The major problem common to assessment of all aspects of neutrophil function is the inherent reactivity of the cells, and thus their behavior may shift significantly due to experimental conditions. Isolation of cells itself represents a stimulus. For example, basal expression of CD11b is increased in isolated neutrophils compared to cells in whole blood and the response to subsequent activation is decreased [13]. In one sense, sensitization during preparation may be useful. In clinical conditions, neutrophils may be primed rather than fully activated, and then the isolation procedure itself may act as a stimulus to which a higher response is seen in patients compared to controls. Given the high degree of activation and increase in flow resistance of isolated cells seen in some clinical studies [66], and that these levels would seem unlikely to be compatible with continued circulation in vivo, it seems that isolation contributes to abnormalities detected in vitro. 
Methods utilizing whole blood or minimal manipulation are highly desirable if one wishes to characterize the in vivo state. Whole blood can be used to measure leukocyte deformability in some filtration devices [29], for analysis of expression of adhesion receptors [13], measurement of cell-cell aggregation [80], and cell morphology with pseudopod formation [60]. Even if an activating stimulus may be present in vivo, the cells may be responding over time after withdrawal of whole blood. In detection of CD11b levels of expression, fixation itself and the period for which cells are held fixed before labeling with fluorescent antibody can markedly influence the intensity of fluorescence. The method of cell handling must therefore be standardized in any series of tests or, for instance, when following changes during a procedure such as cardiopulmonary bypass.

There is a close relationship between neutrophil mechanics, adhesion and activation. It is thus recommended that the state of cell activation be documented in clinical studies since this assists interpretation of rheological studies. As noted above, activation may be assessed from morphology, expression of adhesion molecules and content of F-actin. Other possibilities are to measure the oxidative potential or status of neutrophils using oxidant sensitive dyes or markers of degranulation such as elastase level in plasma. However, there have been relatively few attempts to critically evaluate adhesion methods for clinical studies. Most studies have used increased expression of integrins or loss of L-selectin as indicators of activation and change in adhesive potential. Aggregation of neutrophils or neutrophils with platelets may be easier to standardize, and are better adapted to the use of whole blood than are studies of adhesion to surfaces. While in principle flow-based studies of adhesion yield more detailed and more clearly interpretable results, they require specialized expertise and are seldom used for clinical studies.

\section{Study design and statistical considerations}

\subsection{Reference population and matched controls for clinical studies}

Reference ranges for hemorheological parameters are necessary when used for clinical purposes. These reference ranges should be obtained for each instrument and each parameter using samples from a healthy reference population [49]; an approach for determining clinical chemistry reference ranges may be relevant for hemorheological parameters [3]. Hemorheological parameters are known to be influenced by the following factors: age, sex, ethnicity, obesity, fitness level, smoking, medication, menstrual cycle [51]. Therefore, these factors should be standardized as best as possible. Those diseases known to affect hemorheological parameters [85] should be excluded.

Based on these considerations, samples should be obtained from 20-30 year old subjects with no obvious health problems and with normal hematological indices. They should not smoke or be routinely taking any medication. Separate groups should be formed for male and female subjects. Ethnicity should be standardized if possible or at least mentioned. Fitness level should be taken into account and sedentary subjects should be preferred. Control groups used in clinical studies should be age and sex matched.

All procedures related to the determination of control or reference hemorheological parameters should exactly match the procedures used with patient groups. These include the details of sampling such as tourniquet application, anatomical sampling site and procedures, anticoagulant, time delay between sampling and the actual measurement and sample preparation procedures.

\subsection{Instrument specifications - precision, specificity, sensitivity}

The precision of the method or instrument used in a given study should be tested and reported. This is especially important for custom-built devices and newly developed methods. The precision can be 
assessed as the coefficient of variation (CV) of 10 repeated measurements on the same sample from a healthy control subject [87]. This can be expressed in two ways: (1) CV of replicate measurements of a single sample using the same instrument (i.e., instrument precision, intra-assay variability); (2) CV of 10 measurements on separately prepared samples using the same aliquot of blood (i.e., procedural variability). Obviously, procedural variability includes the variation introduced by the sample preparation procedures (e.g., hematocrit adjustment).

Biological variability of a given hemorheological parameter should be considered as an important determinant of the value of that method or parameter in detecting a "meaningful" difference between two groups (e.g., control and patient groups). Biological variability contributes to the "standardized difference" which can be calculated by dividing the difference between the mean values of the groups by the standard deviation of either group (if the variances of the two groups are homogenous). Alternatively, a "pooled standard deviation" can be used as the denominator which can be calculated as the square root of the sum of the squared standard deviations of the two groups [24]. The standardized difference is a measure of the power of a method or parameter to detect a difference in hemorheological parameters between two groups within a given study design [87]. Alternatively, the standardized difference for a hemorheological parameter can be used to calculate the size of the groups to be able to detect a given difference [87]. While comparing the two hemorheological parameters in terms of their standardized differences, a higher value indicates a higher probability to detect a given difference between the two groups.

The value of hemorheological parameters in clinical studies can be judged by their diagnostic sensitivity and specificity $[36,38,39,85]$. Sensitivity, as a percent, is defined as 100 times the ratio of (true positive findings)/(true positive findings + false negative findings) in a patient group, and is the precision with which a sick person can be identified. Specificity, as a percentage, is defined as 100 times the ratio of (true negative findings)/(true negative findings + false positive findings) in a control or reference group, and is the precision with which healthy subjects can be excluded. Obviously, the merit of any hemorheological parameter will depend upon it having both high sensitivity and specificity.

\section{References}

[1] K.B. Abbitt and G.B. Nash, Rheological properties of the blood influencing selectin-mediated adhesion of flowing leukocytes, Am. J. Physiol. 285 (2003), H229-H240.

[2] A. Acciavatti, D. Pieragalli, T. Provvedi, G.L. Messa, C. Frigerio, M. Saletti, C. Galigani, F. Guideri, M. Franchi, P. Blardi, F.L.L. Pasini and T. Diperri, Circadian variation of platelet-aggregation and blood rheology, Clin. Hemorheol. 13 (1993), $177-186$.

[3] T. Alstrom, R. Grasbeck, M. Hielm and S. Skandsen, Recommendations concerning the collection of reference values in clinical chemistry and activity report, Scand. J. Clin. Lab. Invest. 35 (1975), 1-43.

[4] G. Barshtein, D. Wajnblum and S. Yedgar, Kinetics of linear rouleaux formation studied by visual monitoring of red cell dynamic organization, Biophys. J. 78 (2000), 2470-2474.

[5] V. Bartoli, B. Albanese, P.G. Manescalchi, L. Mannini and G. Pasquini, Influence of blood storage conditions and anticoagulants on results of blood cell filtration test, Clin. Hemorheol. 6 (1986), 137-149.

[6] O.K. Baskurt, Deformability of red blood cells from different species studied by resistive pulse shape analysis technique, Biorheology 33 (1996), 169-179.

[7] O.K. Baskurt, The role of spleen in suppressing the rheological alterations in circulating blood, Clin. Hemorheol. Microcirc. 20 (1999), 181-188.

[8] O.K. Baskurt, T.C. Fisher and H.J. Meiselman, Sensitivity of the cell transit analyzer (CTA) to alterations of red blood cell deformability: role of cell size-pore size ratio and sample preparation, Clin. Hemorheol. 16 (1996), 753-765.

[9] O.K. Baskurt, M.R. Hardeman, M.W. Rampling and H.J. Meiselman (eds), Handbook of Hemorheology and Hemodynamics, IOS Press, Amsterdam, 2008. 
[10] O.K. Baskurt and F. Mat, Importance of measurement temperature in detecting the alterations of red blood cell aggregation and deformability studied by ektacytometry: a study on experimental sepsis in rats, Clin. Hemorheol. Microcirc. 23 (2000), 43-49.

[11] O.K. Baskurt and H.J. Meiselman, Cellular determinants of low-shear blood viscosity, Biorheology 34 (1997), $235-247$.

[12] O.K. Baskurt and H.J. Meiselman, Analyzing shear stress-elongation index curves: comparison of two approaches to simplify data presentation, Clin. Hemorheol. Microcirc. 31 (2004), 23-30.

[13] J. Bateman, S.K. Parida and G.B. Nash, Neutrophil integrin assay for clinical research, Cell. Biochem. Funct. 11 (1993), 87-91.

[14] R.M. Bauersachs, R.B. Wenby and H.J. Meiselman, Determination of specific red blood cell aggregation indices via an automated system, Clin. Hemorheol. 9 (1989), 1-25.

[15] D. Bouix, C. Peyreigne, E. Raynaud, J.F. Monnier, J.P. Micallef and J.F. Brun, Relationships among body composition, hemorheology and exercise performance in rugbymen, Clin. Hemorheol. Microcirc. 19 (1998), 245-254.

[16] K. Breddin, M. Ziemen and O. Bauer, Time and temperature dependent changes of ADP and collagen induced and spontaneous aggregation, Thromb. Res. 19 (1980), 621-638.

[17] P.J.H. Bronkhorst, E.J. Nijhof and J. Sixma, Parametrization of the deformation curve as a tool for standardization and interpretation of ektacytometric measurements, Clin. Hemorheol. 15 (1995), 803-816.

[18] L.M. Butler, H.M. McGettrick and G.B. Nash, Static and dynamic assays of cell adhesion relevant to the vasculature, Methods Mol. Biol. 467 (2009), 211-228.

[19] S.M. Buttrum, G.B. Nash and R. Hatton, Changes in neutrophil rheology after acute ischemia and reperfusion in the rat hindlimb, J. Lab. Clin. Med. 128 (1996), 506-514.

[20] J.A. Chasis and N. Mohandas, Erythrocyte membrane deformability and stability: two distinct membrane properties that are independently regulated by skeletal protein associations, J. Cell Biol. 103 (1986), 343-350.

[21] M.R. Clark, Mean corpuscular haemoglobin concentration and cell deformability, Ann. NY Acad. Sci. 565 (1989), 284294.

[22] M.R. Clark, N. Mohandas and S. Shohet, Osmotic gradient ektacytometry: comprehensive characterization of red cell volume and surface maintenance, Blood 61 (1983), 899-910.

[23] G. Cloutier and Z. Qin, Ultrasound backscattering from non-aggregating and aggregating erythrocytes - a review, Biorheology 34 (1997), 443-470.

[24] J. Cohen, Statistical Power Analysis for the Behavioral Sciences, Lawrance Earlbaum Associates, Hillsdale, NJ, 1988.

[25] G.R. Cokelet, The rheology and tube flow of blood, in: Handbook of Bioengineering, R. Skalak and S. Chien, eds, McGraw-Hill, New York, 1987, pp. 14.1-14.17.

[26] G.R. Cokelet, Viscometric, in vitro and in vivo blood viscosity relationships: how are they related?, Biorheology 36 (1999), 343-358.

[27] P. Connes, N. Nemeth, H.J. Meiselman and O.K. Baskurt, Effect of tourniquet application during blood sampling on red blood cell deformability and aggregation: is it better to keep it on?, Clin. Hemorheol. Microcirc., 2009, in press.

[28] P. Connes, M. Uyuklu, J. Tripette, J.H. Boucher, E. Beltan, T. Chalabi, O. Yalcin, R. Chout, O. Hue, M.D. HardyDessources and O.K. Baskurt, Sampling time after tourniquet removal affects erythrocyte deformability and aggregation measurements, Clin. Hemorheol. Microcirc. 41 (2009), 9-15.

[29] A.M. Cook, S.A. Evans and J.G. Jones, The filterability of leukocytes in undiluted blood, Biorheology 35 (1998), 119-130.

[30] P.R. Craddock, D.E. Hammerschmidt, J.G. White, A.P. Dalmasso and H.S. Jacob, Complement (C5a)-induced granulocyte aggregation in vitro: a possible mechanism for complement-mediated leukostasis and leukopaenia, J. Clin. Invest. 60 (1977), 260-264.

[31] M.S. Diamond and T.A. Springer, A subpopulation of Mac-1 (CD11b/CD18) molecules mediates neutrophil adhesion to ICAM-1 and fibrinogen, J. Cell Biol. 120 (1993), 545-556.

[32] J.G.G. Dobbe, G.J. Steekstra, M.R. Hardeman, C. Ince and C.A. Grimbergen, The measurement of the distribution of red blood cell deformability using an automated rheoscope, Clin. Cytometry 50 (2002), 313-325.

[33] S.C. Donnely, C. Haslett, I. Dransfield, C.E. Robertson, D.C. Carter, J.A. Ross, I.S. Grant and T.F. Tedder, Role of selectins in development of adult respiratory distress syndrome, Lancet 344 (1994), 215-219.

[34] E.A. Evans and B. Kukan, Passive material behaviour of granulocytes based on large deformation and recovery after deformation tests, Blood 64 (1984), 1028-1035.

[35] S. Forconi, Hemorheological changes during venous stasis as result of tourniquet application, Clin. Hemorheol. Microcirc. 20 (1999), 197-199.

[36] S. Forconi, From hyperviscosity to endothelial dysfunction: a return trip?, Clin. Hemorheol. Microcirc. 30 (2009), 155165.

[37] S. Forconi, G. Biasi, M. Guerrini, P. Ravelli, C. Rossi, G. Ferrozzi and S. Pecchi, Arterial and venous blood viscosity in ischemic lower limbs of peripheral obliterative arterial disease patients, J. Cardiovasc. Surg. 20 (1979), 379-384.

[38] S. Forconi and T. Gori, The evolution of the meaning of blood hyperviscosity in cardiovascular physiopathology: should we reinterpret Poiseuille?, Clin. Hemorheol. Microcirc. 42 (2009), 1-6. 
[39] S. Forconi and M. Guerrini, Do hemorheological laboratory assays have any clinical relevance?, Clin. Hemorheol. 16 (1996), 17-22.

[40] S. Forconi, M. Guerrini and D. Pieragalli, Can red cell deformability be measured in clinical medicine?, Clin. Hemorheol. 9 (1989), 27-34.

[41] M.R. Hardeman, P.T. Goedhart, J.G.G. Dobbe and K.P. Lettinga, Laser-assisted optical rotational cell analyzer (LORCA). I. A new instrument for measurement of various structural hemorheological parameters, Clin. Hemorheol. 14 (1994), 605-618.

[42] M.R. Hardeman, P.T. Goedhart and N.H. Schut, Laser-assisted optical rotational cell analyzer (LORCA). II. Red blood cell deformability: elongation index versus cell transit time, Clin. Hemorheol. 14 (1994), 619-630.

[43] M.R. Hardeman and C. Ince, Clinical potential of in vitro measured red cell deformability, a myth?, Clin. Hemorheol. Microcirc. 21 (1999), 277-284.

[44] T.H. Howard and C.O. Oresajo, The kinetics of chemotactic peptide-induced change in F-actin content, F-actin distribution, and the shape of neutrophils, J. Cell Biol. 101 (1985), 1078-1085.

[45] ICSH Expert Panel on Blood Rheology, Guidelines for measurement of blood viscosity and erythrocyte deformability, Clin. Hemorheol. 6 (1986), 439-453.

[46] K.A. Jetha, S. Egginton and G.B. Nash, Increased resistance of neutrophils to deformation upon cooling and rate of recovery on rewarming, Biorheology 40 (2003), 567-576.

[47] J.G. Jones, R.A. Adams and S.A. Evans, Bulk filtration through micropore filters for analyzing blood cell rheology in clinical research, Clin. Hemorheol. 14 (1994), 149-169.

[48] F. Jung, U. Kalus, S. Wolf, G. Pindur and H. Kiesewetter, Influence of sodium fluorescein on erythrocyte aggregation in patients with cerebral microangiopathy, Microvasc. Res. 49 (1995), 246-250.

[49] F. Jung, H. Kiesewetter, H.G. Roggenkamp, H.P. Nuttgens, E.B. Ringelstein, M. Gerhards, G. Kotitschke, E. Wenzel and H. Zeller, Determination of reference ranges of rheologic parameters: study of 653 randomly selected probands of the Aachen district, Klin. Wochenschr. 54 (1986), 375-381.

[50] F. Jung, G. Pindur and H. Kiesewetter, Plasma viscosity dependence on proteins and lipoproteins: Results of Aachen study, Clin. Hemorheol. 12 (1992), 557-571.

[51] F. Jung, H.G. Roggenkamp, E.B. Ringelstein, G. Leipnitz, R. Schneider, H. Kiesewetter and H. Zeller, Effect of sex, age, body weight and smoking on plasma viscosity, Klin. Wochenschr. 64 (1986), 1076-1081.

[52] T.W. Jungi, M.O. Spycher, U.E. Nydegger and S. Barandun, Platelet-leukocyte interactions: selective binding of thrombin stimulated platelets to human monocytes, polymorphonuclear leukocytes and related cell lines, Blood 67 (1986), 629-636.

[53] A.J. Keidan, S.S. Marwah and J. Stuart, Evaluation of phosphate and hepes buffers for study of erythrocyte rheology, Clin. Hemorheol. 7 (1987), 627-635.

[54] A.J. Keidan, M.C. Sowter, S.S. Marwah, C.S. Johnson and J. Stuart, Evaluation of hepes and phosphate buffers for rheological studies of sickle cells, Clin. Hemorheol. 7 (1987), 599-610.

[55] G. Kesmarky, P. Kenyeres, M. Rabai and K. Toth, Plasma viscosity: a forgotton variable, Clin. Hemorheol. Microcirc. 39 (2008), 243-246.

[56] D. Koutsouris, R. Guillet, J.C. Lelievre, M. Boynard, M.T. Guillemin, P. Bertholom, R.B. Wenby, Y. Beuzard and H.J. Meiselman, Individual red blood cell transit times during flow through cylindrical micropores, Clin. Hemorheol. 8 (1988), 453-459.

[57] S.S. Lee, K.H. Ahn, S.J. Lee, K. Sun, P.T. Goedhart and M.R. Hardeman, Shear induced damage of red blood cells monitored by the decrease of their deformability, Korea-Aust. Rheol. J. 16 (2004), 141-146.

[58] G. Lippi, G.L. Salvagno, M. Montagnana, G. Brocco and G.C. Guidi, Influence of the needle bore size used for collecting venous blood samples on routine clinical chemistry testing, Clin. Chem. Lab. Med. 44 (2006), 1009-1014.

[59] L.M. Liu, T.Y. Long and H.J. Xu, Effects of indoor thermal environment on blood viscosity of living creatures, J. Cent. South Univ. Technol. 14 (2007), 86-90.

[60] R.A. Lord and S. Roath, Evaluation and comparison of neutrophil bipolar shape formation with a migration assay, J. Clin. Pathol. 43 (1990), 342-345.

[61] G.D.O. Lowe, Blood rheology in vitro and in vivo, Bailleres Clin. Haematol. 1 (1987), 597-636.

[62] D.A. McCarthy, J. Bernhagen, Y.C. Liu and J.D. Perry, A rapid preparation technique for leukocytes, J. Microscopy 158 (1990), 63-72.

[63] T.G. Mezger, The Rheology Handbook, Oxford Univ. Press, Oxford, 2001.

[64] A.V. Muravyov, V.V. Yakusevich, F.A. Chuchkanov, A.A. Maimistova, S.V. Bulaeva and L.G. Zaitsev, Hemorheological efficiency of drugs, targeting on intracellular phosphodiesterase activity: in vitro study, Clin. Hemorheol. Microcirc. 36 (2007), 327-334.

[65] G.B. Nash, Filterability of blood cells: Methods and clinical applications, Biorheology 27 (1990), 873-882.

[66] G.B. Nash, B. Christopher, A.J.R. Morris and J. Dormandy, Changes in the flow properties of white blood cells after acute myocardial infarction, Br. Heart J. 62 (1989), 329-334. 
[67] G.B. Nash, J.G. Jones, J. Mikita, B. Christopher and J. Dormandy, Effects of preparative procedures and of cell activation on flow of white cells through micropore filters, Br. J. Haematol. 70 (1988), 171-176.

[68] G.B. Nash, J.G. Jones, J. Mikita and J.A. Dormandy, Methods and theory for analysis of flow of white cell subpopulations through micropore filters, Br. J. Haematol. 70 (1988), 165-170.

[69] G.B. Nash and H.J. Meiselman, Rheological properties of individual polymorphonuclear granulocytes and lymphocytes, Clin. Hemorheol. 6 (1986), 87-97.

[70] B. Neu and H.J. Meiselman, Depletion-mediated red blood cell aggregation in polymer solutions, Biophys. J. 83 (2002), $2482-2490$.

[71] F.J. Neumann, I. Ott, M. Gawaz, G. Richardt, H. Holzapfel, M. Jochum and A. Schomig, Cardiac release of cytokines and inflammatory responses in acute myocardial infarction, Circulation 92 (1995), 748-755.

[72] F. Neumann, G. Richardt, M. Schneider, I. Ott, H. Haupt, H. Tillmanns, A. Schomig and B. Rauch, Cardiac release of chemoattractants after iscaemia induced by coronary balloon angioplasty, Br. Heart J. 70 (1993), 27-34.

[73] Z. Pecsvarady, T.C. Fisher, A. Fabok, T.D. Coates and H.J. Meiselman, Kinetics of granulocyte deformability following exprosure to chemotactic stimuli, Blood Cells 18 (1992), 333-352.

[74] D. Pieragalli, A. Acciavatti, C. Galigani, G.L. Messa, P. Blardi, M. Guerrini, S. Forconi and T. Di Perri, Interrelationship between whole blood and washed red cell filterability, Clin. Hemorheol. 7 (1987), 273-276.

[75] M.W. Rampling, H.J. Meiselman, B. Neu and O.K. Baskurt, Influence of cell-specific factors on red blood cell aggregation, Biorheology 41 (2004), 91-112.

[76] O. Raz, O. Rogowski, I. Shapira, N. Maharshak, Y. Karni and S. Berliner, Dissociated effects of physical activity and weight loss on fibrinogen concentrations and markers of red blood cell aggregation. Relevance for life style modification in atherothrombosis, Clin. Hemorheol. Microcirc. 37 (2007), 253-262.

[77] Recommendation for a selected method for the measurement of plasma viscosity, J. Clin. Pathol. 37 (1984), 1147-1152.

[78] H. Relevy, A. Koshkaryev, N. Manny, S. Yedgar and G. Barshtein, Blood banking-induced alteration of red blood cell flow properties, Transfusion 48 (2008), 136-146.

[79] C.S. Rinder, J.L. Bonan, H.M. Rinder, J. Mathew, R. Hines and B.R. Smith, Cardiopulmonary bypass induces leukocyteplatelet adhesion, Blood 79 (1992), 1201-1205.

[80] H.M. Rinder, J.L. Bonan, C.S. Rinder, K.A. Ault and B.R. Smith, Dynamics of leukocyte-platelet adhesion in whole blood, Blood 78 (1991), 1730-1737.

[81] R.F. Ritchie, T.B. Ledue and W.Y. Craig, Patient hydration: a major source of laboratory uncertainty, Clin. Chem. Lab. Med. 45 (2007), 158-166.

[82] V. Saada, T. Cynober, Y. Brossard, P.O. Schischmanoff, A. Sender, H. Cohen, J. Delaunay and G. Tchernia, Incidence of hereditary spherocytosis in a population of jaundiced neonates, Pediatr. Hematol. Oncol. 23 (2006), 387-397.

[83] H. Schmid-Schonbein, H. Rieger and T. Fischer, Blood fluidity as a consequence of red cell fluidity, flow properties of blood an flow behavior in vascular disease, Angiology 31 (1980), 301-319.

[84] H. Schmid-Schonbein, H. Rieger, G. Gallasch and H. Schachtner, Pathological red cell aggregation (clump aggregation). Molecular and electrochemical factors, Bibl. Anat. 16 (1977), 484-489.

[85] S. Spitzer, H. Kiesewetter, F. Jung, R. Bach and H. Schieffer, Guidelines for the determination of haemorheological parameters and the performance of clinical therapeutic trials, Clin. Hemorheol. 11 (1991), 657-662.

[86] G.J. Streekstra, Quantification of the fraction of undeformable red blood cells in a cell population using ektacytometry, in: The Deformation of Red Blood Cells in a Couette Flow, Dissertation, Univ. Utrecht, The Netherlands, 1994, pp. 55-68.

[87] J. Stuart, P.C.W. Stone, G. Freyburger, M.R. Boisseau and D.G. Altman, Instrument precision and biological variability determine the number of patients required for rheological studies, Clin. Hemorheol. 9 (1989), 181-197.

[88] G.B. Thurston and N.M. Henderson, Viscoelasticity of human blood, in: Handbook of Hemorheology and Hemodynamics, O.K. Baskurt, M.R. Hardeman, M.W. Rampling and H.J. Meiselman, eds, IOS Press, Amsterdam, 2007, pp. $72-90$.

[89] M. Uyuklu, M. Cengiz, P. Ulker, T. Hever, J. Tripette, P. Connes, N. Nemeth, H.J. Meiselman and O.K. Baskurt, Effects of storage duration and temperature of human blood on red cell deformability and aggregation, Clin. Hemorheol. Microcirc. 41 (2009), 269-278.

[90] M. Uyuklu, H.J. Meiselman and O.K. Baskurt, Effect of hemoglobin oxygenation level on red blood cell deformability and aggregation parameters, Clin. Hemorheol. Microcirc. 41 (2009), 179-188.

[91] E. Volger, H. Schmid-Schonbein, J. Gosen, H.J. Klose and K.A. Kline, Microrheology and light transmission of blood. IV. The kinetics of artificial red cell aggregaton induced by Dextran, Pflugers Arch. 354 (1975), 319-337.

[92] M.P. Wautier, W. El Nemer, P. Gane, J.D. Rain, J.P. Cartron, Y. Colin, C. Le van Kim and J.L. Wautier, Increased adhesion to endothelial cells of erythrocytes from patients with polycythemia vera is mediated by laminin alpha 5 chain and Lu/BCAM, Blood 11 (2007), 894-901. 